\title{
SERRATUS-INTERCOSTAL PLANE BLOCK FOR REDUCTION MAMMOPLASTY IN A CHILD PATIENT
}

Sandra Fernandez-Caballero, Maria Perez-Herrero.

Department of Anesthesiology, Hospital Clinico Universitario de Valladolid, Spain.

\section{BACKGROUND AND AIMS}

Pubertal gynecomastia is a bilateral or unilateral enlargement of breast tissue in male, starting at age of 10 . The most frequent etiology is idiopathic and an important factor is the disparity between testosterone and estradiol levels that produces transient estradiol excess.

We present a case of a child with idiopathic gynecomastia and a successful serratusintercostal plane block that was executed for reduction mammoplasty.

\section{METHODS}

A 13 year-old-boy, with medical history of asthma, was scheduled for reduction mammoplasty. In the operating room, standard monitoring was placed and general anesthesia was induced with fentanyl $1 \mathrm{mcg} / \mathrm{kg}$, propofol $1 \mathrm{mg} / \mathrm{kg}$ and rocuronium $0.6 \mathrm{mg} / \mathrm{kg}$. After the orotracheal intubation, serratusintercostal plane block (Fig.1), was performed under ultrasound vision, using a $50 \mathrm{~mm}$ needle. $10 \mathrm{ml}$ of $0.25 \%$ bupivacaine was administered (Fig.2). There were no complications associated with the procedure.

Once the patient was extubated, he was admitted to the post-anesthesia care unit (PACU), with good control of the pain.

\section{RESULTS}

In PACU, the patient had a Visual Analog Scale (VAS) score of 0 , not requiring any analgesia. No complications were registered.

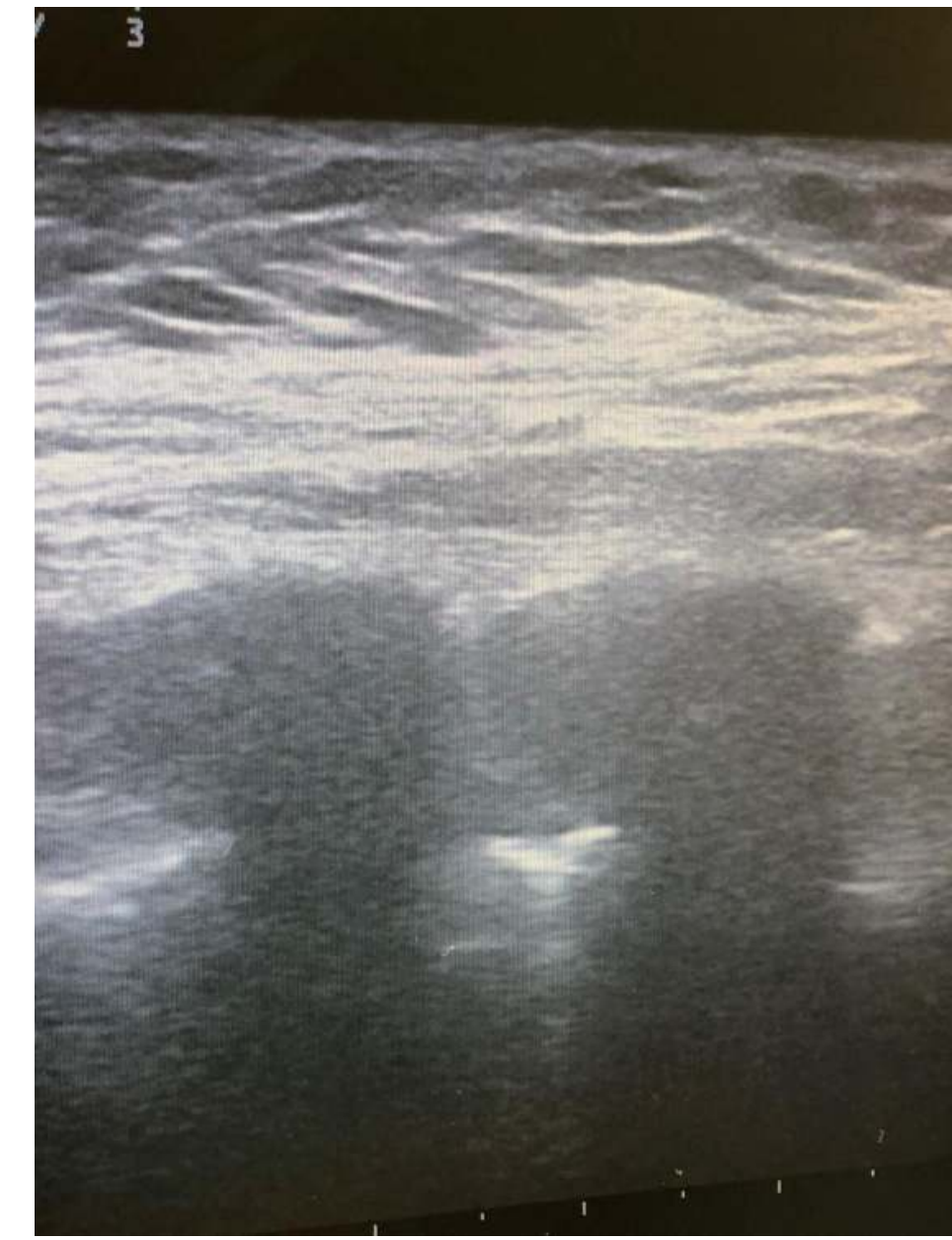

(Fig.1) Serratus-intercostal plane block

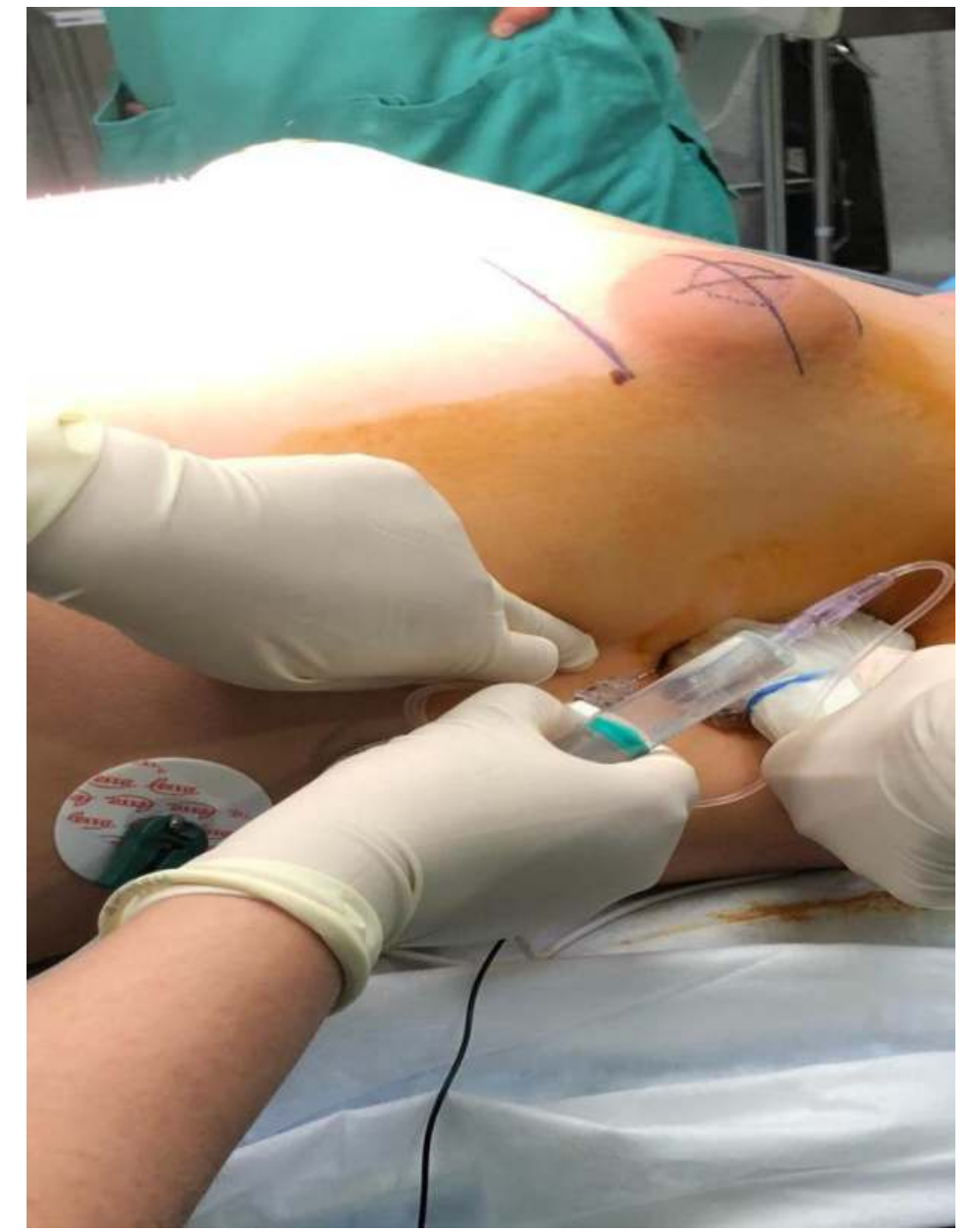
was administered
(Fig.2) Bupivacaine

Children are considered high risk for difficult pain control. Serratus-intercostal plane block has been proved to be useful in opioid-free anesthesia.

As we know, this is the first serratusintercostal plane block described in children. We found good analgesic results.

\section{CONCLUSIONS}

Serratus-intercostal plane block guarantees an excellent perioperative analgesia, in multimodal and opioid-free anesthesia protocols. No side effects were found. 